The MacLean Center for Clinical Medical Ethics at the University of Chicago Pritzker School of Medicine J D Lantos

Southwestern Medical Center at Dallas, Texas, USA JE Tyson

Grace Maternity Hospital, Halifax, Nova Scotia, Canada

A Allen

Department of Pediatrics, Center for Medical Ethics, University of Pittsburgh, Philadelphia, USA

J Frader

Case Western Reserve University, Cleveland, Ohio, USA

$M$ Hack

University of Tennessee Medical Group, Memphis,

Tennessee, USA

$S$ Korones

The Children's Hospital, Denver Colorado, USA G Merenstein

Michigan State University, Michigan, USA

N Paneth

Milton Hershey Medical Center, Hershey, USA $R$ Poland

McMaster University, Hamilton, Ontario, Canada

S Saigal

Department of Neonatology, Stanford Medical Center, California, USA D Stevenson

Harvard University Joint Program in Neonatology, Children's Hospital, Boston, Massachusetts, USA R Truog

L Van Marter

Correspondence to: Dr John D Lantos, La Rabida Hospital and Research Center, Research Center, Lake Michigan, Chicago, II Lake Michiga

\section{Withholding and withdrawing life sustaining treatment in neonatal intensive care: issues for the 1990s}

\author{
John D Lantos, Jon E Tyson, Alexander Allen, Joel Frader, Maureen Hack, \\ Sheldon Korones, Gerald Merenstein, Nigel Paneth, Ronald L Poland, Saroj Saigal, \\ David Stevenson, Robert D Truog, Linda J Van Marter
}

The transition from fetus to newborn is accompanied by dramatic physiological changes in the infant and by equally profound changes in emotional and moral attitudes, as well as changes in legal status among parents, caregivers, and society. The fetus becomes a baby, adults cross the border to parenthood, and medical responsibility shifts from the obstetrician to the paediatrician. These radical changes create challenges even when the baby is healthy. But when the baby is critically ill, and decisions need to be taken quickly, the medical complexities amplify the moral, legal, and psychological concerns.

During the 1970s, ethical deliberations about appropriate personal and societal responses to critically ill newborns led to widespread agreement ${ }^{12}$ (though not complete consensus $^{34}$ ) that decisions should primarily reflect the interests of the infant. Interests of parents, other family members, or society at large were secondary. Infants' interests might best be served by continuing or foregoing treatment. If the decision was made to forego treatment there was no legal or moral difference between withholding and withdrawing treatment. ${ }^{5}$ Consensus on these issues helped secure medical treatment for newborns with disabling conditions, such as Down's syndrome or meningomyelocele.

The 1980s brought a more restrictive view of ethical options which would have prohibited discontinuation of treatment unless infants were either comatose or the treatment considered 'futile' or 'inhumane'. 6 These proposals were controversial, partly because concepts such as futility or inhumanity were vague and difficult to put into practice, and also because they went against the intuitions of parents and medical professionals. ${ }^{7}$ In the United States attempts by the Reagan administration to enforce these ethical guidelines created an atmosphere of distrust and fear. ${ }^{8}$ Careful study and comparison of different approaches to end of life care for neonates became difficult.

At the same time advances in neonatology tended to magnify the dilemmas, especially with respect to extremely low birthweight infants, and created a new set of issues for which guidelines from the 1970s were difficult to apply. ${ }^{9} 10$ Furthermore, changes in societal attitudes and public policies regarding treatment decisions for dying adults created an increasing disparity in the way these decisions were handled for patients of different ages. ${ }^{11}$ While adults were gaining a 'right to die' that included the right to forego fluid and nutrition, the parents of neonates were losing the right to forego all but the most inefficacious treatments.

\section{Methods}

To reopen discussion of the issue of withholding and withdrawing treatment in neonatal intensive care we asked a group of paediatricians, neonatologists, and intensive care physicians to describe cases in which they or their colleagues would consider withholding or withdrawing life sustaining treatment. All of these physicians were familiar with the discussions about ethical issues in neonatology over the past two decades. In May 1993 we brought these physicians together to review the cases, and to determine whether they thought a decision to forego treatment would have been morally appropriate.

Thirty five cases were submitted. Most belonged to one of three categories. The first comprised cases of extreme prematurity. At the borderline of viability, decisions had to be made whether to initiate treatment in the delivery room or to discontinue treatment after a very brief period of life support.

The second group comprised cases in which infants had developed severe, chronic problems for which life prolonging treatment was possible but for which the outcome was uncertain and the burdens of treatment high. In such cases the issue was generally not disagreement between families and professionals. Rather, both had difficulty determining what was best for a particular infant, and worked together to reach decisions.

The final category consisted of cases in which there was intractable disagreement between doctors and parents about the appropriateness of further treatment. In most of these cases doctors felt that death was inevitable in spite of 
continued treatment, but parents insisted on treatment being continued. In some instances parents requested that treatment be withdrawn and physicians insisted that treatment continue. Representative cases from each category are presented as follows.

EXTREME PREMATURITY AND DECISIONS IN THE PERINATAL PERIOD

Case 1

A 30 year old woman presented with ruptured membranes at an estimated gestational age of 22 weeks. A Gram stain of the amniotic fluid showed Gram positive cocci in chain formation. Because of the estimated gestational age and the presumed infection with group B streptococci, the parents were informed that survival was impossible. Labour was induced without fetal monitoring. When delivery occurred, the baby was gasping in room air and had Apgar scores of 3 at one minute and 1 at five minutes. A paediatrician was called. She intubated the baby and began mechanical ventilation. The infant quickly achieved oxygen saturations of $90-95 \%$ on relatively low ventilator settings. The parents had initially requested that treatment be discontinued, but, on seeing how well the baby was doing, changed their minds. The baby did well for 10 days, then developed a large intracranial haemorrhage. After discussion with the parents treatment was discontinued.

\section{Case 2}

A 21 year old woman presented with ruptured membranes at an estimated gestational age of 21 weeks. Delivery occurred four hours later. There was no discussion with the mother regarding treatment options before delivery. The paediatrician attending the delivery assessed the infant to be between 21 and 23 weeks. Birth weight was 540 g. Apgar scores were 1 at one and five minutes. No oxygen or mechanical ventilation were provided. The infant died shortly after birth.

\section{Case 3}

A 23 week old, $498 \mathrm{~g}$ girl was born to a 21 year old mother with premature rupture of membranes and chorioamnionitis. Although given the option of withholding life support, the parents requested that everything be done. Over the first weeks of life, the baby had severe metabolic derangements, staphylococcal and candida sepsis, bilateral grade III intraventricular haemorrhages, numerous pneumothoraces and a patent ductus arteriosus. In spite of physicians' recommendations that treatment be stopped, parents continued to request aggressive treatment, although they agreed to a Do Not Resuscitate order. By 3 months of age, the baby was receiving 30\% oxygen, a combination of enteral and parenteral feeds, and her parents were pleased with her progress and making plans to take her home.
Comments - Many academic medical centres and organisations of paediatricians are currently developing guidelines for the treatment of extremely premature and low birthweight neonates. For example, The Canadian Pediatric Society comes out against treatment for babies whose gestational age is under 23 completed weeks, recommends that parents be given the option of treatment for babies whose gestational age is 23-24 completed weeks, and strongly recommends continued treatment for babies whose gestational age is 25 or more completed weeks. ${ }^{11 a}$

Such guidelines reflect the general principle that the treatment of newborns is obligatory if it offers a reasonable chance of survival (with the possible exception of situations in which survival is possible only with severe medical or neurodevelopmental sequelae). When chances for survival are low, treatment may be withheld or withdrawn. In applying these principles, theoretical debate focuses on two issues. First, how accurately can we predict survival for a particular baby? Second, assuming that we can predict prognosis, what constitutes a reasonable chance of survival?

Assessment of prognosis for an infant of very low birth weight relies on estimated gestational age, birth weight, and the clinical status of the infant. None of these factors can be precisely established before birth. Despite rigorous dating of the pregnancy, gestational age estimates are often off by one to three weeks. Furthermore, birth weight varies widely for babies of the same gestational age. For example, at 24 weeks' gestation, birth weights range from 400-1000 g (Harvard Medical School Joint Program in Neonatology; unpublished data). As a result, it is difficult to make definite plans for treatment or non-treatment until the baby is born, and the neonatologist has a chance to assess size, maturity, and clinical status.

Furthermore, the chances of survival for an infant at a given birth weight or gestational age differ depending on race, sex, the philosophy of obstetric and neonatal care, and other unknown factors. ${ }^{12}$ Thus non-treatment policies which seek to standardise decision making for infants with a similar prognosis must allow for wide local variation.

Focus on predicted survival may oversimplify the real issues, for several reasons. Precise prognoses are necessarily artificial in the context of any particular case. In the real world the chances for survival are always difficult to determine and may change rapidly over time. Thus estimates are valuable only as a way of distinguishing types of clinical situations and changes in moral obligations at the extremes of the spectrum of prognosis. In virtually all cases a decision whether to initiate or forego treatment can only be made after the paediatrician or neonatologist assesses the newborn and determines whether the neonate's initial condition is consistent with the prenatal estimates of gestational age.

Because it is so difficult to give precise prognostic assessments prenatally, communication between obstetricians, neonatologists, 
or paediatricians and parents is essential to avoid misleadingly absolute predictions of viability or non-viability. Prenatal decisions to initiate or withhold treatment should be reconsidered if the newborn is either more or less viable than anticipated. Shared and dynamic decision making, which mirrors the process for older children or competent adults, ensures that decisions incorporate parental values, the infant's interests, and an optimal understanding of the facts and the inherent uncertainties in the child's clinical situation.

Prognosis will always depend on a combination of factors, and will always be somewhat uncertain for any particular baby. ${ }^{13}$ Currently, however, survival rates for babies under 24 weeks' gestation or less than $600 \mathrm{~g}$ birth weight are predictably poor enough so that parental choices regarding resuscitation of such babies ought to be encouraged and respected. Decisions to respect parental choices in these situations acknowledge that in order for parents to choose to forego life sustaining treatment on their child's behalf, survival does not have to be impossible or unprecedented - it only has to be very unlikely. Prognostic inaccuracy and changing technological potential both preclude an attempt to define a boundary of probability that is, $5 \%, 10 \%$ of some other per cent chance of survival - for withholding treatment more precisely.

In situations where there is no time to involve parents in decision making (when a woman presents in premature labour and delivery is imminent), the presumption should generally be in favour of treatment unless survival is unprecedented.

\section{CHRONIC DISEASE AND BURDENSOME TREATMENT \\ Case 4}

A baby weighing $1100 \mathrm{~g}$ developed severe bronchopulmonary dysplasia, requiring prolonged assisted ventilation. Four attempts at extubation failed. He was finally weaned from the ventilator at 11 months of age, but quickly developed respiratory distress and was reintubated. At the age of 12 months, his cognitive function was at the nine month level. By the age of 17 months, he was weaned from the ventilator for a few weeks, but required intensive respiratory treatment and was failing to thrive. His respiratory physician recommended long term home ventilation. His parents, who were very involved in his care, requested that he not be placed back on a ventilator and that he be allowed to die.

Case 5

A full term newborn with arthrogryposis and omphalocele continued to require assisted ventilation after repair of the omphalocele. After being told that the baby would be quadriplegic and suffer repeated painful bone fractures, her parents opted to discontinue ventilator support. Unexpectedly, the infant did not die. At age 2, she remains quadriplegic but cognitively intact, and is developing normal language skills.

Comment - In these cases there was some chance of long term survival, but survival would most likely have required prolonged dependence on medical technology. The children would also be left with severe disabilities. In both cases cognitive function was nearly intact.

Individual physicians within our group disagreed about whether, under the circumstances, they would have recommended continuation of treatment. All agreed, however, that in such cases it would be morally permissible for parents and physicians together to decide to withdraw treatment. A number of factors supported these decisions.

The children's suffering was a primary concern. Long term ventilator treatment for a toddler may require sedation. Suctioning may be painful. A life of constant respiratory distress and air hunger may be a life of constant fear and anxiety. In these circumstances to consider only the chances for survival was seen as inhumane.

Concerns were also raised about the effect of continued treatment on the parents and other family members. ${ }^{14}$ Such concerns included not only the economic cost of care, but the emotional demands of having a child whose care entails a series of medical crises and who requires ongoing constant attention and concern. When treatment will clearly lead to the child's survival and recovery, such considerations pale in relation to the obligations to do what is best for the sick child. However, when it is unclear whether continued treatment is in the child's interest, a more complex calculus of family interests may be necessary. ${ }^{15} 16$

Legally, treatment decisions rest with the parents until they have been judged neglectful by a court of law. Such judgments rely on conclusions that parents are not acting in the child's best interest. In these cases doctors could not agree about the value of continued treatment or about whether stopping treatment was an obvious or egregious violation of the child's interest. Even if a physician felt that a parental decision was unacceptable the proper response would not be to override parental wishes unilaterally. Instead, it would be to notify parents that the law requires physicians to seek a determination by child protection agencies of whether the parents' request to discontinue treatment was permissible. Such an approach would correctly relocate the conflict as one between parents and that state's interest in child protection, rather than as a conflict between parents and paediatricians.

Such difficult assessments can best be made by people who have a long term relationship with the child. Thus parents, in consultation with a physician who has provided ongoing care to the child, are in the best position to make the difficult decision to discontinue support. Paediatricians must be parents' allies in difficult cases. Optimal decision making is facilitated by the involvement of a primary physician who is highly knowledgeable, consistently involved in the care of the child, and who develops ongoing rapport with parents. Too often, such children fall through 
the cracks of the primary care system as their problems are so complicated that they require prolonged tertiary care. In tertiary care settings attending physicians change periodically, and no one may have primary responsibility for the ongoing management of chronic medical problems and ethical decision making.

The discussion raised interesting and seldom addressed questions about informed consent in paediatrics. In situations involving chronic disease and burdensome treatment parents may request that treatment be withdrawn. If such parents find a physician who agrees that withdrawal is appropriate, babies are allowed to die. Other physicians find withdrawal unacceptable and may not heed parental requests to discontinue treatment. Variations in physicians' practice styles and moral values can lead to an arbitrariness in available options for parents.

Physicians should be careful to separate their personal views, which may be in favour of continued treatment, from current medical, legal, and moral standards of care for such children. These standards give parents the right to discontinue treatment unless this right has been overruled by a judge. Parents should be informed of these rights. Physicians in such situations must try to help parents understand the complexities of determining prognosis, and the changing nature of prognostic estimates over time, and may thus recommend continuing treatment. They should also inform parents when discontinuation of treatment is permissible.

\section{INTRACTABLE DISAGREEMENT \\ Case 6}

A newborn weighing $750 \mathrm{~g}$ with an estimated gestational age of 24 weeks developed severe respiratory distress syndrome. $\mathrm{He}$ also had transient renal failure, seizures on the second day of life, and prolonged direct hyperbilirubinaemia. He subsequently developed severe bronchopulmonary dysplasia, was ventilated for 55 days, and remained oxygen dependent for 80 days. The parents frequently expressed concerns about his quality of life and questioned whether intensive care should be continued. Physicians felt that prognosis for neurodevelopmental outcome was uncertain and recommended continued treatment. The parents were hesitant, but agreed to ligation of a patent ductus arteriosus and insertion of a central venous line. Treatment was continued and he was discharged home at 133 days of age. At $4 \frac{1}{2}$ years of age, the child is microcephalic, mentally retarded, cortically blind and has quadriplegic cerebral palsy. $\mathrm{He}$ is entirely dependent on his caretaker. His parents feel bitter that their repeated requests for treatment to be discontinued were ignored.

\section{Case 7}

A girl weighing $589 \mathrm{~g}$ was born at 23 weeks' gestation. Apgar scores were 1 at one minute and 5 at five minutes. She developed severe respiratory distress, electrolyte imbalances, hyperglycaemia, necrotising enterocolitis and bilateral intraventricular haemorrhages with ventricular dilation. Neonatologists recommended discontinuation of treatment. Although the mother seldom visited, when she did, she insisted that everything be done to keep her baby alive. The father showed little interest in either visiting or participating in treatment decisions. Maximal ventilatory support was continued until the infant died on day 15.

\section{Case 8}

A full term baby, born after a pregnancy complicated by oligohydramnios, developed cyanosis, grunting, and retractions shortly after birth. He was intubated at 10 minutes. In spite of maximal ventilatory support, $\mathrm{pCO}_{2} \mathrm{~s}$ remained in the 90-100 range with the $\mathrm{pH}$ values between 6.99 and $7 \cdot 11$. A presumptive diagnosis of pulmonary hypoplasia was made and doctors recommended discontinuation of treatment. Parents requested transfer to another hospital for a second opinion. Although the physicians felt this was unnecessary, they arranged the transfer. At the other hospital, a renal ultrasound scan revealed dysplastic kidneys and the infant was removed from the ventilator with parental approval.

Comment - Participants agreed that cases of intractable disagreement were relatively rare but emotionally trying. There were two types of cases. First, those in which parents insisted on treatment that physicians felt would not be beneficial. Discussion of these cases are part of the ongoing debate over moral obligations in cases where treatment seems to be futile. ${ }^{17}$ Second, those in which parents strongly opposed recommendations for continued treatment, but eventually acquiesced. These cases are the type that have received the most coverage in the lay press, ${ }^{18}$ and have recently been addressed. ${ }^{19}$

The most difficult 'futility' cases in neonatology are those in which the parents seem to be emotionally unattached to the child but, nevertheless, demand continued treatment. In such cases, providers often feel called on to protect infants from the pain and suffering of useless overtreatment demanded by inconsiderate or even malevolent parents. In theory, physicians and other providers have the right to withdraw from a case or refuse to provide treatment which they view as morally objectionable. However, the realities of most clinical situations preclude the physicians from exercising this option.

Four options are currently available to the physician in these circumstances. First, the physician can continue to treat, allowing his or her own moral values to be outweighed by the parents' demands to continue life support. Second, the physician could unilaterally refuse to provide treatment and insist that parents find another physician. Legal concerns about abandonment would dictate that the physician help the parents locate another physician. Third, the physician could seek a judicial determination that continued painful and nonbeneficial treatment constitutes a form of child 
abuse and should be withdrawn. However, courts generally rule in favour of continued treatment in such cases. Finally, institutional policies defining futile care could preclude or reduce the need for ad hoc decision making regarding individual cases. Policies could either be substantive - mechanical ventilation should not be provided to patients in a persistent vegetative state; or procedural - treatment may be withdrawn over parental objections if an ethics committee recommends it.

Cases of intractable disagreement, where physicians want to continue treatment and parents want to stop, raise different issues. These cases illustrate the discrepancies between the rights that adults have to refuse medical treatment and the rights that parents have in regard to their children. Decisions for neonates have life long impact on patients and their families, while neonatologists are generally involved with families for only a brief period of time. This longer term perspective may temper enthusiasm for treatments of uncertain efficacy, or those which are likely to leave children with severe long term morbidity.

\section{costs}

There was heated disagreement within our group about the appropriateness of using estimates of the cost of treatment to guide policies regarding decisions to forego life sustaining treatment. The unique vulnerability of newborns was reflected in opinions that cost considerations should not be used only or primarily for allocation decisions in neonatology, but should compare the cost effectiveness of neonatal care with treatments used for patients at other stages of life. In many public policy debates neonatal care is singled out for rationing in a way that does not reflect the relative cost effectiveness of neonatal care compared with intensive care for the elderly. ${ }^{20}$

There was also concern that costs are an artificial and somewhat arbitrary figure, reflecting varying degrees of technological advances, inept administrative procedures, inefficient use of resources, and greed among health care professionals and lawyers. Elimination of these extraneous factors would decrease costs considerably and may decrease the pressure to end lives because the bills are unmanageable.

The urgency of economic considerations varied widely, with Canadian physicians having a different view than US physicians, and physicians in public hospitals having a different view than those in private hospitals. Even those who recognised a need for fiscal accountability had difficulty with proposals that would withhold treatment from particular patients based on that patient's projected expenses. These discussions showed how any attempt to ration must consider deeply held professional moral imperatives which forbid the withholding of accessible medical treatment, based on abstract notions of justice or theoretical econometric models.

\section{Conclusion}

Treatment withdrawal dilemmas in neonatal intensive care arise primarily at two points in the life of a neonate. First, decisions must be made whether to initiate treatment for extremely premature babies. These decisions reflect parental motivations, obstetric assessments of maternal risks, and evolving prognostic predictions of the likelihood of neonatal morbidity or mortality. Guidelines for deciding when babies should be considered non-viable, when intensive care is optional, or when intensive care is obligatory, should reflect recent hospital specific data on morbidity and mortality for babies at different birth weights and gestational ages.

Shared decision making among obstetricians, neonatologists, and parents is essential. Ethics committees have been helpful in describing general guidelines for decision making, but are rarely consulted for day to day decision making. They may be most useful in developing institutional policies for dealing with categories of patients, such as the extremely premature babies, or in helping to arbitrate intractable disagreements.

Tough decisions also arise for neonates who have survived the first days but who have severe congenital or iatrogenic problems. In these cases the probability of survival, the burdens of treatment, the likelihood of major morbidity, and the impact of decisions on families must all be considered.

Disagreement about decisions to withhold or withdraw treatment in neonates no longer centres on disagreement about moral principles or the types of broad guidelines which should guide such decisions. Instead, a widespread consensus has emerged that the child's interests are the central moral consideration. Other considerations are relevant only when it is unclear whether continued treatment is in the child's interest.

However, the concept of best interest is only precise enough to get us so far. In particular cases 'best interest' may be evaluated differently by lawyers, judges, physicians, parents, philosophers or special interest advocacy groups. $^{21}$ These differences will not be resolved by enunciating more finely tuned or revised principles, but by showing how moral principles must be supple enough to accommodate ever changing clinical realities.

Practical guidance for decision making will emerge as general principles are tested through analysis of their applicability to particular cases. Guidelines, while crucial, will never be sufficient, because they will always define not only areas of right and wrong action but also areas of ambiguity. In such ambiguous areas, we will need to interpret guidelines by paying attention to the informed intuitions of parents, professionals, and concerned citizens who must respond to particular babies in particular situations. This report attempts a first step in that direction. The Center for Clinical Medical Ethics is supported by grants
from the Henry J Kaiser Family Foundation, the Andrew W Mellon Foundation, and the Pew Charitable Trusts. The Mellon Foundation, and the Pew Charitable Trusts. The
conference on which this paper is based was supported by a conference on which this paper is
grant from the Harris Foundation. 
1 Jonsen A. Critical tissues in newborn intensive care: A conference report and policy proposal. Pediatrics 1975; 55: confere $756-85$

2 President's Commission for the Study of Ethical Problems in Medicine and Biomedical and Behavioral Research. Deciding to forego life-sustaining treatment. In: Seriously ill newborns. Washington, DC: US Government Printing Office, 1983: 197-229.

3 Duff R, Campbell AGM. Moral and ethical dilemmas in the special care nursery. $N$ Engl $f$ Med 1973; 289: $890-4$.

4 Todres ID, Krane D, Howell MC, Shannon DC. Pediatricians' attitudes affecting decision making in defective newborns. Pediatrics 1977; 60: 197-201.

5 Robertson JA, Fost N. Passive euthanasia of defective newborn infants: legal considerations. $\mathcal{F}$ Pediatr 1976; 88: newbor

6 Amendments to the Child Abuse Prevention and Treatment and Adoption Reform Act. Public Law 1984; 98-457.

7 Kopelman LM, Irons TG, Kopelman AE. Neonatologists judge the 'Baby Doe' regulations. $N$ Engl $f$ Med 1988; 318: $677-83$.

8 Lantos JD. Baby Doe five years later: implications for child health. $N$ Engl f Med 1987; 317: 444-7.

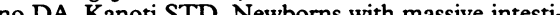
nal loss: Difficult choices. $N$ Engl $\mathcal{f}$ Med 1988; 318: nal los:

10 Lantos JD, Frader J. Extracorporeal membrane oxygenation and the ethics of clinical research in pediatrics. $N$ Engl $\mathcal{F}$ Med 1990; 323: 409-13.

11 Wanzer SH, Federman DD, Adelstein SJ, et al. The physician's responsibility towards hopelessly ill patients. A second look. N Engl f Med 1989; 320: 844-9.

11a Fetus and Newborn Committee, Canadian Pediatric Society; Maternal Fetal Medicine Committee, Society of Obstetricians and Gynecologists of Canada. Management of the women with threshold birth of an infant of extremely low gestational age. Can Med Assoc $\mathcal{F}$ 1994; 151: 547-53.

12 Hack M, Fanaroff AA. Outcomes of extremely-lowbirth-weight infants between 1982 and 1988. $N$ Engl $f$ Med 1989; 321: 1642-7.

13 Fischer AF, Stevenson DK. The consequences of uncertainty. An empirical approach to medical decision making in neonata! intensive care. $¥ A M A$ 1987; 258: 1929-31.

14 Hardwig J. What about the family? Hastings Cent Rep 1990; 20: 5-11.

15 Strong C. The neonatologist's duty to patients and parents. Hastings Cent Rep 1984; 14: 1-4.

16 Lantos JD, Kohrman AF. Ethical aspects of pediatric home care. Pediatrics 1992; 89: 920-4.

17 Schneiderman LJ, Jecker N. Futility in practice. Arch Intern Med 1993; 153: 437-41.

18 Stinson A, Stinson P. The long dying of baby Andrew. Atlantic Monthly Press: New York, 1984.

19 Harrison $H$. The principles for family-centered neonatal care. Pediatrics 1993; 92: 643-50.

20 Hadorn DC. Setting health care priorities in Oregon. Costeffectiveness meets the rule of rescue. $¥ A M A 1991 ; 265$ effectiven

21 Doyal L, Wilsher D. Towards guidelines for withholding and withdrawal of life-prolonging treatment in neonatal medicine. Arch Dis Child 1994; 70: F66-70. 